\title{
INVESTIGACIÓN SOBRE EL COTIDIANO DEL SUJETO: OPORTUNIDADES PARA UNA CIENCIA APLICADA
}

\author{
Manuel Amezcua ${ }^{1}$, Sandra Milena Hernández Zambrano ${ }^{2}$
}

\footnotetext{
${ }^{1}$ Máster en Investigación en Ciencias Socio-Sanitarias. Profesor de Documentación e Informática Clínicas. Facultad de Ciencias de la Salud, Universidad de Granada, España. E-mail: mamezcuam@ugr.es

${ }^{2}$ Licenciada en Enfermería. Grupo de Investigación en Salud Comunitaria INVESCOM, Fundación Index, Granada, España. E-mail: samyta599@hotmail.com
}

\begin{abstract}
RESUMEN: Desde un modelo de investigación emergente, la Investigación Aplicada a los Cuidados, se propone reflexionar sobre una de las áreas de indagación: la Investigación sobre el Cotidiano del Sujeto. El cotidiano del sujeto se conceptualiza como un espacio de interacción social donde las personas ejercen autonomía para tomar decisiones y ejecutar acciones para cuidar su salud. A través de diseños principalmente cualitativos, la Investigación sobre el Cotidiano del Sujeto aporta evidencias útiles y pertinentes para la práctica enfermera porque hablan del contexto y desde la perspectiva de los sujetos. Estas evidencias pueden encuadrarse en cuatro grandes dimensiones: la comprensión del padecimiento humano ante la enfermedad, la cultura del cuidado, la percepción sobre las intervenciones en salud, y el cotidiano del cuidador. Finalmente se plantean algunos desafíos que nos propone la Investigación sobre el Cotidiano del Sujeto e implican tres dimensiones imprescindibles en el ejercicio del cuidado: la dimensión ética, política y científica. DESCRIPTORES: Investigación en enfermería. Gestión del conocimiento para la investigación en salud. Tendencias. Investigación cualitativa.
\end{abstract}

\section{PESQUISA SOBRE O COTIDIANO DO SUJEITO: OPORTUNIDADES PARA UMA CIÊNCIA APLICADA}

RESUMO: A partir de um modelo de pesquisa emergente, a Investigação Aplicada ao Cuidado, pretende refletir sobre uma das suas áreas de investigação: a Investigação sobre o Cotidiano do Sujeito. O cotidiano dos sujeitos é conceituado como um espaço de interação social onde as pessoas exercem autonomia para tomar decisões e implementar ações para se manter saudável. Através de desenhos principalmente qualitativos, a Investigação sobre o Cotidiano do Sujeito fornece provas úteis e relevantes para a prática de enfermagem porque falam do contexto e da perspectiva dos sujeitos. Essas evidências podem ser agrupados em quatro grandes dimensões: a compreensão da condição humana à doença, a cultura do cuidado, a percepção das intervenções de saúde, e do cotidiano do cuidador. Finalmente, surgem alguns desafios evocados pelo Investigação sobre o Cotidiano do Sujeito a partir de três dimensões essenciais no exercício do cuidado: ética, política e ciência.

DESCRITORES: Pesquisa em enfermagem. Gestão do conhecimento para a pesquisa em saúde. Tendências. Pesquisa qualitativa.

\section{DAILY RESEARCH SUBJECT: OPPORTUNITIES FOR AN APPLIED SCIENCE}

\begin{abstract}
From a model of emerging research, the Applied Research to Care, proposes to reflect about one of its areas of inquiry: Research about the Daily Life of the Subject. The daily life of the subject is conceptualized as a social interaction space where the subjects exercise autonomy to make decisions and implement actions to stay healthy. Through primarily qualitative designs, Daily Life of the Subject provides useful and pertinent evidence for nursing practice because they speak from the context and perspective of the subjects. This evidence can be classified into four broad categories: understanding the human condition prior to disease, the culture of care, the perception of health care, and the daily caregiver. Finally, some challenges emerge from Daily Life of the Subject that we categorize in the three main dimensions in the execution of care: ethics, politics, and science.
\end{abstract}

DESCRIPTORS: Nursing research. Knowledge management for health research. Trends. Qualitative research. 


\section{INTRODUCCIÓN}

Las políticas en investigación y los modelos de gestión del conocimiento en salud corroboran el dominio de la investigación básica sobre la investigación aplicada. La primacía de la investigación positivista en el campo de las ciencias de la salud requiere la emergencia de nuevas propuestas de investigación de corte socio-humanista que dirijan la mirada a la comprensión del sujeto en su entorno cotidiano. La preocupación por el sujeto en el contexto del cuidado se hace visible en el creciente interés por modelos teóricos que son llamados por las enfermeras en un intento de clarificar los dilemas inherentes a la intersubjetividad, necesariamente presentes en el proceso cuidador. Pensadores de muy diversa procedencia teórica como Foucault, Bourdieu, Beck, Douglas, Benner, Freire, por mencionar solo algunos, vienen siendo citados de forma recurrente en los estudios de enfermería de corte socio-sanitario publicados en la última década, en un intento de búsqueda de horizontes teóricos que iluminen una praxis centrada en las necesidades del sujeto.

Entre los modelos propiamente enfermeros, resulta de obligada mención, la corriente transculturalista liderada por Leininger y continuada por Purnell, Spector, Siles y otros, que pone énfasis en el cuidado del otro (la interacción con el otro culturalmente diferente). ${ }^{1}$ Por su parte, los seguidores de la Teoría de la Adaptación (Roy, Peplau, Rogers o Levine), subrayan el sentido dialógico del cuidado de enfermería. El propio modelo ha evolucionado significativamente en su concepción de la persona, que ha trascendido desde la descripción individual a su dimensión colectiva. ${ }^{2}$

El maridaje que en las últimas décadas se está produciendo entre la investigación en enfermería y las metodologías cualitativas está permitiendo profundizar en dimensiones de la salud poco estudiadas hasta este momento, como son las afectaciones del padecimiento, las influencias del contexto cultural, la inequidad de género, el cuidado familiar, etc. De forma progresiva, aunque débilmente aún, encontramos propuestas investigadoras que se apartan de la tradicional concepción del enfermo como objeto de atención, para ocuparse del paciente-persona, situando al sujeto en el eje del proceso cuidador. Entre las propuestas recientes podemos destacar la de Enders y cols. sobre la Ciencia-Acción, que enfatiza el análisis crítico de la práctica profesional con una propuesta transformadora a partir de la reflexión durante la acción. ${ }^{3}$ Valverde por su parte propone un modelo de relación centrado en los significados del paciente, una relación de ayuda que utiliza la narrativa para dar nuevos significados, contenido y perspectiva a la experiencia del enfermar. ${ }^{4}$ Recuperar y sistematizar la experiencia del cuidado para generar conocimiento, y adoptar una práctica rutinaria con sentido esencialmente transformador que mejore la calidad del cuidado cotidiano en los servicios de salud, se han planteado entre los principales desafíos para incrementar el cuerpo de conocimientos de la disciplina enfermera. ${ }^{5}$

En la misma línea se sitúan algunos grupos de investigación vinculados a la Fundación Index (Granada, España), que a través del enfoque de la Investigación Aplicada a los Cuidados (IAC) nos proponen reflexionar sobre las relaciones ontológicas de un tipo de investigación que pone énfasis en la condición del sujeto y sus complejidades. ${ }^{6}$ Este modelo sugiere tres direcciones o grandes áreas donde se construye el conocimiento: (a) investigación sobre el cotidiano del sujeto, (b) investigación sobre resultados en salud, y (c) investigación sobre prácticas basadas en Evidencias Científicas. ${ }^{6}$

Este artículo reflexiona sobre la primera de ellas, la Investigación sobre el Cotidiano del Sujeto (ICS), quizá la más compleja de conceptualizar pero a la vez la que más claridad ha de aportar sobre la esencia de una investigación aplicada en el ámbito de la Enfermería. Y lo haremos a partir de cinco cuestiones: ¿qué consecuencias ha traído la supremacía del modelo positivista en la investigación de las ciencias de la salud?, ¿cómo se conceptualiza la ICS?, ¿qué características definen esta área de investigación?, ¿cómo se construye conocimiento enfermero y cómo ha sido la producción científica de las enfermeras en la ICS?, y finalmente ¿qué desafíos tendría la ICS en el ejercicio de cuidado?

\section{Positivismo e investigación en salud}

Cuando imaginamos el quehacer científico del investigador de bata blanca, en un laboratorio aséptico, controlado y ajeno al contexto de los sujetos, recordamos al estereotipo del investigador positivista de la modernidad. El entorno aséptico que representa su laboratorio está cargado de símbolos y de un conjunto de rituales propios del método experimental. El orden, el control, lo esterilizado son elementos simbólicos que "marcan la distancia entre los sujetos que investigan y los fenómenos observados para dar apariencia de objetividad" .7:729 
En la actualidad, la creciente influencia de la investigación biomédica y su vertiente tecnológica, refleja en el campo de las ciencias de la salud la supremacía del investigador de bata blanca, el dominio de la investigación básica sobre la investigación aplicada. Algunos ejemplos de este modelo hegemónico son los sistemas de clasificación del conocimiento, que están fundamentados en los principios positivistas de las ciencias. La UNESCO, por ejemplo, clasifica los conocimientos de las ciencias clínicas, la epidemiología, y la medicina forense con mayor jerarquía que los conocimientos emergentes de la antropología, la psicología o la sociología. Otro ejemplo es el movimiento de la Práctica Basada en Evidencias, que jerarquiza las "pruebas" a partir de las investigaciones de corte experimental. Y otra muestra es que las investigaciones sobre biotecnología celular y molecular o farmacología de corte experimental tienen mayor prioridad a la hora de asignación de recursos.

En el campo de los cuidados de la salud, acentuar la mirada en el paradigma positivista implica la reproducción de un modelo hegemónico que margina o incluso excluye el conocimiento proveniente de la dimensión social, cultural y subjetiva inherente al género humano. Como señala Beltrán, "al seleccionar lo relevante se desprecia lo irrelevante, que queda sin estudiar y desaparece del ámbito del conocimiento al no constituirse como objeto científico: toda selección comporta una ocultación". 8:44

Si bien se reconoce que la vinculación entre práctica e investigación bajo la corriente positivista es la que ha generado hasta hoy las evidencias que mayormente sirven de base para la práctica de la enfermería, ${ }^{9}$ también representa límites significativos en la construcción del conocimiento enfermero. Por un lado limitan la generación de evidencias cualitativas que aportan conocimientos útiles, pertinentes y contextuales para mejorar la calidad de vida de los ciudadanos y las colectividades. Por otro lado detienen el crecimiento disciplinar de la enfermería, que en buena medida se alimenta de conocimientos provenientes de las ciencias sociales y humanísticas. La ciencia de la enfermería requiere construirse en la constante y dinámica interrelación con los sujetos: sus experiencias vividas, sentidos y significados compartidos, sus expectativas y valores, su cultura y su historia.

La primacía de la investigación positivista en el campo de las ciencias de la salud requiere la emergencia de nuevas propuestas de investigación de corte socio-humanista. Es el caso de la corriente denominada Investigación Aplicada a los Cuidados (IAC), que la Fundación Index viene fomentando desde hace más de dos décadas. La IAC se define como un proceso de generación de conocimiento basado en metodología científica dirigido a la mejora o conservación de la salud de las personas, desde el respeto a su manera de sentir y de vivir y a sus posibilidades de participación efectiva. ${ }^{6}$ El fin último de la IAC es proporcionar mejores niveles de salud a un sujeto libre y pensante, con estatus de ciudadano, y ello se consigue desplazando el laboratorio de investigación al escenario de lo cotidiano. ${ }^{6}$

\section{El cotidiano como área de indagación}

¿Cómo se conceptualiza la Investigación sobre el Cotidiano del Sujeto?, ¿qué características definen esta área de investigación? Se reconoce a la Escuela de Frankfurt como introductora de la crítica a la racionalidad del paradigma positivista. Si trasladamos sus consecuencias prácticas al campo de las ciencias de la salud, la reducción de la relación con los sujetos (pacientes) a la dimensión biológica, fisiopatológica, cuantificable y observable, se vería compensada con el paradigma constructivista, que reivindica al ser humano como centro de discusión. El área de ICS está ubicada en este paradigma constructivista y específicamente en el ámbito teórico de la microsociología, que se ocupa del comportamiento social cotidiano. ${ }^{10} \mathrm{El}$ estudio de tales formas de interacción social es una de las áreas más absorbentes de la investigación sociológica. ${ }^{10}$ Schütz, Luckmann, Heller y Habermas, como precursores del paradigma constructivista, han aportado elementos conceptuales desde la corriente fenomenológica, la teoría marxista o la teoría de la acción comunicativa, que no son excluyentes sino que se enriquecen en la complementariedad y que argumentan la relevancia de la vida cotidiana como objeto de investigación.

Alfred Schütz define el mundo de la vida cotidiana como la región de la realidad en la que el hombre puede intervenir y puede modificar mientras opera en ella. Este mundo público que describe Schütz, lo define como un mundo intersubjetivo, circundante, y comunicativo y es el ámbito de la intersubjetividad donde afirma se encuentra el espacio propio de la vida cotidiana. ${ }^{11}$ Agnes Heller toma como marco de referencia el modelo marxista para hablar de las cuestiones históricas, materiales e institucionales de la vida cotidiana. ${ }^{12}$ Heller define la vida cotidiana como un hecho social, en tanto que el actor se reproduce a sí mismo como actor social 
y genera nuevas condiciones para la reproducción de la sociedad. La reproducción de la realidad en la vida cotidiana, ilumina aspectos significativos de los sistemas e instituciones sociales más amplias, es decir, nos permite comprender modelos de pensamiento de la sociedad que reproducen los sujetos en su entorno cotidiano. ${ }^{10} \mathrm{La}$ autora afirma que la vida cotidiana es esencialmente histórica y que el estudio de la vida cotidiana es un análisis de la acción e interacción social. ${ }^{12}$

Jürgen Habermas propone, a través de la teoría de la acción comunicativa, transitar de un modelo monológico fundamentado en la racionalidad instrumental y ajeno al sujeto, hacia un modelo dialógico fundamentado en la intersubjetividad. Con ello quiso enfatizar el sentido comunicativo discursivo como fuente de saberes, afirmando que la objetividad no se garantiza por la distancia o abstención valorativa, sino que se garantiza por los resultados de la discusión a partir de diferentes puntos de vista. ${ }^{13} \mathrm{Y}$ todas aquellas valoraciones, razones y sentires se debaten en el mundo de la vida o mundo cotidiano. También el referente foucaultiano invita a proyectar la mirada crítica sobre lo cotidiano, problematizándolo. Su visión del poder remite a las relaciones entre las personas en un espacio de convivencia donde se desarrollan todos los acontecimientos de la vida humana, donde tienen lugar los discursos y las prácticas cotidianas. ${ }^{14}$

Es pues a partir de diversas corrientes de pensamiento, entre ellas la fenomenología, la teoría marxista, la teoría de la acción comunicativa, o el posestructuralismo, pero todas ellas provenientes de las ciencias sociales y las humanidades, que encontramos elementos comunes y complementarios que nos permiten comprender la complejidad e importancia del cotidiano del sujeto como área de investigación.

Pero ¿Cuáles son estos elementos comunes?, $¿$ Las particularidades del lugar donde se aproximan las expectativas y los padeceres de la gente? Veamos algunas características del cotidiano del sujeto:

- es cambiante y emergente, es decir, siempre produce o reproduce nuevas estructuras de pensamiento, ya que las realidades son cambiantes y los sujetos son cambiantes también. Esto confirma que la vida cotidiana no es rutinaria, trivial, natural, banal, habitual, usual, frecuente, acostumbrada, poco relevante o cíclica. Estas definiciones que tradicionalmente asociamos a lo cotidiano, menosprecian la riqueza e importancia de este espacio de interacción.
- se alimenta en la intersubjetividad. No es una mirada al sujeto como individuo, sino a cómo se relacionan los sujetos en el entorno cotidiano. Por eso, como refiere Heller, requiere un análisis de la acción e interacción social. ${ }^{12}$

- en las acciones de la vida cotidiana se muestra la conexión entre lo individual y lo social. Ilumina aspectos significativos de los sistemas e instituciones sociales más amplias, es decir, nos permite comprender modelos de pensamiento de la sociedad que reproducen los sujetos en su entorno cotidiano. ${ }^{10}$

- posibilita la acción y transformación de la realidad. Es un espacio de autonomía donde los sujetos ejercen poder, donde toman decisiones y transforman su realidad. Habermas enfatiza esa acción transformadora del mundo de la vida. ${ }^{13}$

A partir de estas características y en el marco de referencia de la IAC, podemos conceptualizar el cotidiano del sujeto como un espacio de interacción social donde los sujetos ejercen autonomía para tomar decisiones y ejecutar acciones para cuidar su salud. El sujeto reproduce estructuras de pensamiento condicionadas por su contexto y produce estructuras de pensamiento condicionadas por sus valores, experiencias y conocimientos. Pero es en su entorno cotidiano donde esta información es reconfigurada para construir un esquema de referencia y concretar un marco de acción.

Cuando hablamos de Investigación sobre el Cotidiano del Sujeto nos referimos a un proceso de construcción de conocimiento cuyo objeto de estudio es la vida cotidiana de las personas que cuidamos. Lo cotidiano "delimita un espacio compartido donde aparentemente no pasa nada, pero que en realidad es donde ocurre todo: la salud, la enfermedad, el padecimiento, la necesidad, el cuerpo, la familia, la relación terapéutica, el cuidado, la cultura." ${ }^{14: 729} \mathrm{El}$ objetivo de esta área de investigación es aportar evidencias que generen procesos de comprensión y transformación de la realidad de los sujetos que cuidamos para mejorar su calidad de vida. Para construir este conocimiento se requiere de la interacción continua entre los sujetos cuidados y los sujetos cuidadores, y esto se posibilita a través de la acción comunicativa.

\section{Metodologías emergentes en la ICS: cómo se construye conocimiento enfermero}

¿Cómo se construye conocimiento enfermero desde la IAC como perspectiva de investigación, y más concretamente desde la ICS? El cotidiano 
del sujeto como materia de investigación sugiere metodologías emergentes del paradigma constructivista, coherentes con "la naturaleza simbólica, sustantiva, interpretativa, histórica y dialéctica"15:18 del objeto de estudio. La IAC reivindica su naturaleza humanística poniendo su punto de mira en la comprensión del padecimiento humano, dirigiéndose a las respuestas del sujeto y teniendo en cuenta el contexto: las relaciones sociales que se producen en torno al proceso salud-enfermedadatención-salud y la diversidad de significados culturales que están presentes. ${ }^{6}$ Es así que los métodos tradicionalmente empleados en la ciencia positivista resultan altamente reduccionistas a la hora de comprender las realidades implicadas en el cotidiano del sujeto.

Cuando se problematiza sobre el cotidiano del sujeto, es la investigación cualitativa la que marca la hoja de ruta metodológica para obtener una visión holística, comprensiva y contextualizada de la realidad. Schütz y Habermas, precursores del concepto de la vida cotidiana o mundo de la vida como objeto de estudio, han aportado las bases epistemológicas para el desarrollo de metodologías de tipo cualitativo como la fenomenología y la Investigación Acción Participativa. La importancia de la validez de sus tesis se sienta en las bases intersubjetivas del mundo social de la vida cotidiana y en los fundamentos de análisis de la realidad social. ${ }^{12}$ También nosotros sugerimos que en la investigación sobre el cotidiano del sujeto hay una dominancia de los enfoques constructivistas, de la etnografía, de la fenomenología y la hermenéutica, lo que abre oportunidades para el desarrollo de metodologías cualitativas en el ámbito de la salud. ${ }^{6}$

Si consultamos bases de datos bibliográficas como CUIDEN o LILACS encontraremos algunos trabajos que aluden al mundo cotidiano del paciente, lo que indica que las enfermeras iberoamericanas han iniciado una incipiente producción científica sobre esta área de investigación, con la excepción de la realidad brasileña, donde se concentra la mayor parte de la producción, con un notorio recorrido. La mayoría de los estudios son de naturaleza cualitativa, que se preguntan por diferentes dimensiones del cuidado en el cotidiano del sujeto, utilizando principalmente diseños fenomenológicos, etnográficos, de investigación participativa, o biográficos, que se realizan sobre diferentes grupos poblacionales: mujeres y adolescentes que padecen VIH/SIDA, ancianos que padecen trastornos mentales, Diabetes Mellitus tipo II o Insuficiencia Renal Crónica, pacientes con Insuficiencia Cardíaca, personas que padecen tuberculosis, niños y mujeres víctimas de violencia, mujeres gestantes, mujeres en climaterio, por citar solo algunas líneas de investigación que están presentes.

\section{Las evidencias de la ICS}

Aceptar que la ICS logre aportar evidencias científicas válidas puede resultar un asunto polémico. Ya hemos apuntado que el conocimiento proveniente de esta área de investigación procede fundamentalmente de diseños cualitativos, que son relegados a los niveles menos recomendables por los sistemas de jerarquización del conocimiento propuestos por el movimiento de la Práctica Basada en Evidencias. Por ello debemos reiterar la necesidad de superar las visiones reduccionistas propias del positivismo y ampliar el horizonte de lo pensable y de lo posible. De esta forma, cuando revisamos investigaciones publicadas en el contexto iberoamericano que consideran como objeto de estudio el cotidiano del sujeto, identificamos evidencias que podemos clasificar a partir de cuatro grandes categorías. Sólo a efectos ilustrativos, incluiremos en cada una de ellas algunos ejemplos de estudios publicados en los últimos años, si bien la revisión se ha realizado sobre toda la producción identificada en las anteriormente mencionadas bases de datos, hasta saturar las categorías.

a) Comprensión del padecimiento ante la enfermedad. El padecimiento es entendido como enfermedad en primera persona y hace referencia a la experiencia cultural, interpersonal y personal (subjetiva) de la enfermedad. Se refiere a las afectaciones que la enfermedad produce al paciente y su familia. ${ }^{16} \mathrm{El}$ conocimiento generado a partir de la ICS nos aporta evidencias para comprender cómo los sujetos vivencian el padecimiento, cómo perciben la enfermedad, sus angustias, sus incertidumbres, sus sentimientos y la manera como afrontan sus adversidades en su entorno cotidiano.

La fenomenología, las historias de vida, los relatos biográficos, son enfoques de investigación que por su naturaleza se acercan a la comprensión de la experiencia humana en primera persona. Es a partir de este conocimiento que se marcan itinerarios para abordar un cuidado más cercano a las realidades de quienes los vivencian, se identifican recursos adaptativos, se comprenden las repercusiones de la enfermedad sobre el cotidiano y de los tratamientos en la calidad de vida de los sujetos. 
A través de este enfoque, se ha verificado que la ostomía genera una serie de alteraciones en la vida de su portador ocasionando transformaciones en el cotidiano del sujeto y afectando no solo su estructura física, sino también aspectos psicológicos y sociales como su capacidad de adaptación o las relaciones sociales. A través de este estudio los autores resaltan la importancia de la familia y las relaciones sociales como los principales puntos de apoyo y seguridad de los pacientes ostomizados. ${ }^{17}$ Desde una perspectiva fenomenológica, se ha profundizado en la forma en que el padecimiento de las personas que han sufrido un infarto agudo de miocardio genera importantes cambios en su estilo y calidad de vida, afectando este proceso no solo a la persona que lo padece sino también a los miembros de su familia, por ello propone incluir en el plan de cuidados a los cuidadores familiares. ${ }^{18}$ Desde las narrativas, una informante relata cómo sus vivencias cotidianas en torno al padecimiento del cáncer contribuyen a que los profesionales de enfermería comprendan el sufrimiento desde las necesidades sentidas del paciente y se sensibilicen ante la necesidad de humanizar el sistema sanitario y considerar el apoyo familiar de los pacientes y sus creencias. ${ }^{19}$

b) Cultura del cuidado. Se refiere a las evidencias que describen los conocimientos, creencias, valores y prácticas de cuidado de los sujetos en su entorno cotidiano. El distanciamiento entre el discurso científico de los profesionales y los saberes populares conlleva necesariamente choques culturales en la relación profesional-paciente. Los saberes populares representan cosmovisiones y portan símbolos e imaginarios sobre cómo los sujetos mantienen y recuperan la salud y previenen la enfermedad. Por tanto las evidencias provenientes de las investigaciones sobre la cultura del cuidado nos sirven para "conocer la realidad desde otra perspectiva, la que tiene que ver con la interpretación que hace el propio sujeto en su contexto social (dimensión emic), para diferenciarla de nuestras interpretaciones como profesionales o como investigadores adoctrinados por nuestro propio saber científico (dimensión etic)" . 16:61

Los métodos etnográficos, por su naturaleza descriptiva y contextual y por el énfasis en la comprensión de los sentidos y significados culturales, se nos muestran como idóneos en este apartado de la ICS. Los profesionales de la salud, cuando sitúan el padecimiento en el contexto social y cultural del paciente, pueden elaborar planes de cuidados culturalmente congruentes con la realidad de quienes lo vivencian.
A la luz de este grupo de evidencias se ha desarrollado un modelo de cuidado de enfermería que integra a las mujeres-abuelas y su familia para la promoción, protección y apoyo a la lactancia materna en el cotidiano familiar. ${ }^{20}$ Este modelo muestra la importancia de sumergirse en las distintas culturas para intercambiar, negociar y reflexionar sobre las prácticas culturales del amamantamiento. Cada mujer del estudio amamantaba a sus hijos de acuerdo a su contexto histórico, su experiencia y la experiencia con la práctica de la lactancia materna. ${ }^{20}$ También se han identificado mediante un estudio etnográfico las maneras en que las personas expresan sus emociones durante la espera en los servicios de urgencias, encontrando que son espacios impersonales que limitan la comunicación y el acompañamiento entre las personas, y hacen que se generen diferentes estados emocionales que se expresan a través del cuerpo. ${ }^{21}$ Este estudio permitiría implementar, en las instituciones de salud, mecanismos para favorecer la comunicación asertiva entre usuarios y personal de salud, para la toma de decisiones oportunas y adecuadas a cada situación, de manera que la atención de urgencias sea un proceso humanizado. ${ }^{21}$

c) Percepción sobre las intervenciones en salud. La Investigación Evaluativa de tipo cualitativo permite conocer la percepción que los sujetos tienen sobre los tratamientos e intervenciones que reciben desde los servicios de salud. Este tipo de investigación sitúa el laboratorio en la propia realidad, profundiza la visión de los sujetos y los vincula como actores participantes del proceso de investigación. En el campo de la salud comunitaria Swanson destaca la importancia del enfoque cualitativo en la investigación evaluativa, que proporciona evidencias sobre los mecanismos en los que se basan las intervenciones exitosas, examina el proceso de implementación de un programa de atención en salud y no solo mide los resultados. ${ }^{22}$

A través de un estudio cualitativo sobre el padecimiento de las personas afectadas por diabetes, se ha descrito cómo una sociedad hipermedicalizada contribuye a un manejo infectivo del régimen terapéutico. Los pacientes diabéticos dan mucha más importancia al tratamiento farmacológico que a los autocuidados y les cuesta asimilar la cronicidad. Los resultados de este estudio alertan a los profesionales de enfermería hacia la necesidad de promocionar el autocuidado, el tratamiento no farmacológico y los estilos de vida saludables. ${ }^{23}$ Bajo esta misma línea metodológica y con el objeto de identificar cómo los pacientes que 
padecen tuberculosis se adhieren a su tratamiento, un estudio logra extraer del discurso de estos pacientes los elementos del día a día que precisaban la atención de los profesionales, y que concretaban en tres dimensiones: los elementos objetivos en relación con la racionalidad del diagnóstico, tratamiento, orientaciones, signos y síntomas, y falta de recursos; los elementos subjetivos derivados de las emociones (miedos, aislamiento, tristeza, desesperanza, problemas familiares, etc.); y la influencia de la espiritualidad y la fe para tratar la enfermedad. ${ }^{24}$ Sobre este tema, Martins y cols. afirman que "la adhesión al tratamiento se produce cuando el individuo se apropia del tratamiento, es decir, cuando hay un compromiso derivado de la comprensión del verdadero significado de la terapia y su eficacia". ${ }^{25: 117}$ En su estudio sobre la adhesión al tratamiento antirretroviral en escolares, identifica algunos elementos que facilitan el proceso de adhesión y resaltan la importancia del papel de la enfermera en la promoción de la autonomía, el autocuidado y del propiciar la participación de los niños en la toma de decisiones relacionadas con su enfermedad y tratamiento. ${ }^{25}$

d) El cotidiano del cuidador. Cuando las enfermeras convierten su quehacer en un ejercicio rutinario, monótono y acrítico, que resta importancia a las relaciones que construyen diariamente con los pacientes, se limita la posibilidad de generar evidencias que hablen de la interacción de la enfermera con los pacientes y su entorno. Por contra, ya Foucault alertó sobre la necesidad de poner el punto de mira en el día a día de los gestos sin aparente importancia, de convertir el universo de las inter-subjetividades en escenario de investigación, de problematizar con todo lo que transita en la sombra. ${ }^{14}$ Por tanto la práctica cotidiana de la enfermera es una fuente inacabable de experiencias que posibilita el desarrollo de una investigación acorde a la realidad donde se ejerce el cuidado.

En una unidad oncológica, al analizar la percepción de las enfermeras sobre el significado del proceso de cuidar al paciente con cáncer, se aprecia que, a pesar del sufrimiento y la sensación de impotencia ante la muerte, los profesionales son capaces de producir sentimientos de gratificación, ligados a pequeñas acciones (un gesto de cariño, un pequeño roce) con efectos muy positivos sobre el bienestar del paciente. ${ }^{26}$ En el campo de la salud mental y ante una reforma psiquiátrica que transforma la concepción de la locura en la sociedad en Brasil, un estudio describe las estrategias utilizadas por los enfermeros de familia en el cuidado a las personas con trastorno psicológico, resaltando su desconocimiento acerca del término "sufrimiento psíquico" o "trastorno mental" y por tanto de los cuidados inherentes a los pacientes con trastornos psicológicos. ${ }^{27}$

Si las evidencias provenientes del cotidiano de la enfermera marcan rutas para abordar un cuidado que hable de las interacciones pacienteenfermera, también es necesario considerar otros actores que están presentes en el escenario cotidiano del cuidado. El cuidado familiar se entiende como aquella prestación de cuidados a personas dependientes por parte de familiares, amigos y otras personas de su red social que no reciben retribución económica por la ayuda que ofrecen. Numerosos estudios demuestran que son los miembros de la familia los que con mayor frecuencia prestan este tipo de cuidado. La familia y los cuidadores desempeñan un papel importante en el mantenimiento, protección y recuperación de la salud, no solo por el cuidado a las personas dependientes sino por ser un núcleo generador de hábitos. ${ }^{28} \mathrm{El}$ conocimiento generado a partir del cotidiano del cuidador contribuye a establecer relaciones de cooperación, coordinación y coresponsabilidad entre los profesionales sanitarios y las familias para el ejercicio del cuidado.

Un estudio del contexto en el que se desenvuelven los cuidados de familiares con demencia avanzada, constata que las cuidadoras familiares se han acostumbrado a llevar una vida restringida y con poco margen para el desarrollo personal. Una situación que las enfermeras pueden mejorar a través de asesoramiento, sensibilización y búsqueda de equidad en las prácticas cuidadoras. ${ }^{29}$ En el mismo campo de las demencias, otro estudio analiza cómo los cuidadores familiares identifican, denominan e interpretan el padecimiento y cómo establecen y desarrollan estrategias de cuidados. Los resultados enfatizan la necesidad de legitimar el saber que disponen los familiares en cuanto son cuidadores experimentados. ${ }^{30}$

\section{CONSIDERACIONES FINALES}

Las investigaciones focalizadas en el cotidiano de los sujetos aportan conocimientos útiles, pertinentes y contextuales para la toma de decisiones en el cuidado de la salud. Por tanto, generan evidencias útiles y pertinentes para la práctica enfermera porque hablan del contexto y desde la perspectiva de los sujetos. Los diseños utilizados en esta área de investigación son generalmente de corte cualitativo, que proporcionan conocimien- 
tos cercanos a la realidad de los ciudadanos. De esta forma, la ICS converge con la investigación cualitativa para mostrarse como un instrumento poderoso para establecer las bases de acción del cuidado y promover el desarrollo disciplinar de la enfermería. ${ }^{22}$

Son muchos y muy sugerentes los desafíos que nos propone la ICS, que podríamos segmentar a partir de tres dimensiones imprescindibles en el ejercicio del cuidado: la ética, la política y la científica. La dimensión ética hace referencia a la acción comunicativa como puente de interacción esencial que abre paso a una relación dialógica y humanizada entre las enfermeras y los ciudadanos. La fuente principal de provisión de bienestar en la vida diaria en el marco familiar, ha posibilitado la construcción de una nueva cultura política del cuidado basada en la corresponsabilidad, ${ }^{31}$ que sería la dimensión política que referíamos. Mientras que los desafíos en la dimensión científica deben concentrarse en la generación e implementación de evidencias cualitativas, tan denostadas hasta ahora por la ciencia positiva.

Sería deseable que las políticas de investigación fueran acordes con la necesidad de ampliar notablemente el conocimiento sobre el mundo cotidiano del paciente, sobre sus estilos de vida y sobre las afectaciones inmediatas de la enfermedad, con una visión integral e integradora que involucre al paciente de forma activa. ${ }^{6}$ La corriente aquí desarrollada de Investigación sobre el Cotidiano del Sujeto marca la ruta de una parcela científica con un fuerte componente humanístico sobre la que ya puede considerarse existe un importante acervo de conocimiento. El que tenga su reflejo en las prioridades de investigación a la hora de asignar recursos dependerá de las voluntades de quienes marcan las políticas en salud.

\section{REFERENCIAS}

1. Fornons Fontdevila D. Madeleine Leininger: claroscuro trascultural. Index de Enfermería. 2010; 19(2-3):172-6.

2. Oliveira TC, Silva VM, Lopes MVO, Araujo TL. Análisis evolutivo del concepto persona en la teoría de adaptación de Roy. Cultura de los Cuidados. 2007; XI(22):64-9.

3. Enders BC, Ferreira PBP, Monteiro AI. A Ciênciaação: fundamentos filosóficos e relevância para enfermagem. Texto Contexo Enferm. 2010 Jan-Mar; 19(1):161-7.

4. Valverde C. Enfermería centrada en los significados del Paciente ${ }^{\circledR}$. Un modelo basado en la narrativa y en la ética del otro. Index de Enfermería. 2008; 17(3):157-8.

5. Turisi MLV. Cuidar e investigar: desafíos metodológicos en enfermería. Texto Contexto Enferm. 2011; 20(1):175-83.

6. Amezcua M. Investigación aplicada en cuidados de salud. Index de Enfermería. 2010; 19(4): 237-39.

7. García C. Entre ciencia y vida cotidiana: el cuidado de las personas como objeto de conocimiento. En: Cristobal Torres, editor. Sociología y realidad social: libro homenaje a Miguel Beltrán. Madrid (ES): Centro de Investigaciones Sociológicas; 2008; p. 729.

8. Beltrán M. Ciencia y sociología. Madrid (ES): Centro de Investigaciones Sociológicas; 2001.

9. Landeros Olvera E, Salazar González B, Cruz Quevedo E. La influencia del positivismo en la investigación y práctica de enfermería. Index Enfermería. 2009; 18(4): 263-66.

10. Hernández Tezoquipa I, Arenas Monreal ML, Valde Santiago R. El cuidado a la salud en el ámbito doméstico: interacción social y vida cotidiana. Rev Saúde Pública. 2001; 35(5):443-50.

11. Schütz A, Luckmann T. La estructura del mundo de vida. Buenos Aires (AR): Amorrortu, 1997.

12. Estrada Saavedra M. La vida y el mundo: distinción conceptual entre mundo de vida y vida cotidiana. Sociológica. 2000; 15(43):103-51.

13. Hoyos Vasquez G. Las ciencias de la discusión en la teoría del actuar comunicativo. En: Elster J, Inglehart R, Eisler R, editores. Reflexiones sobre la investigación en ciencias sociales y estudios políticos. Bogotá (CO): Universidad Nacional de Colombia, 2003; p. 113-30.

14. Amezcua M. Foucault y las enfermeras: pulsando el poder en lo cotidiano. Index Enfermería. 2009; 18(2):77-9.

15. Triviño Z, Sanhueza O. Paradigmas de investigación en enfermería. Cienc Enfermería. 2005; XI(1): 17-24.

16. Amezcua M. Enfermedad y padecimiento: significados del enfermar para la práctica de los cuidados. Cultura de los Cuidados. 2000; IV(7-8): 60-7.

17. Gomes AMS, Vasconcelos MA, da Silva RCC, Neto FRGX. Sentimentos e expectativas de sujeitos com estomia à luz do modelo conceitual de Levine. Enfermería Comunitaria [en línea]. 2011[access 2011 Dic 4]; 7(1).Disponible en http:/ / www.index-f. com/comunitaria/v7n1/ec7627.php

18. Solano M, Siles J, Fernandes G. Vivencias de las esposas cuyas parejas han sufrido un infarto agudo de miocardio. Un estudio fenomenológico. Index Enfermería. 2008; 17(1): 7-11.

19. León Cruz MJ. Como se vive un problema de salud desde la fe. El relato de una paciente de su enfermedad. Archivos de la Memoria [en línea]. 2008 [access 2011 Dic 5]; 5(2):. Disponible en http:/ / www. index-f.com/memoria/5/a20819.php 
20. Teixeira MA, Nitschke RG, Gasperi P, Siedler MJ Significados de avós sobre a prática do aleitamento materno no cotidiano familiar: a cultura do quererpoder amamentar. Texto Contexto Enferm. 2006; 15(1):98-106.

21. Madrigal Ramírez MC, Lopera Betancur MA, Forero Pulido C, García Henao L. El cuerpo: silencioso pero significativo en expresión. Av. Enferm. 2011; XXIX(I):67-74

22. De la Cuesta C. La contribución de la evidencia cualitativa al campo del cuidado y la salud comunitaria. Index Enfermería. 2005; 14(50):47-52.

23. Urdaniz Ruiz C, Hueso Navarro F, Pórtoles Chueca S, Santacruz Redrado EM. El Padecimiento de personas afectadas de Diabetes Tipo 2, explicación del "Manejo Inefectivo del Régimen Terapéutico". Rev Paraninfo Digital [en línea]. 2010 [access 2011 Dec 5]; 9. Disponible en: http:/ / www.index-f.com/ para/n9/i026.php

24. Silva AMFB, Mello FCQ, Figueiredo NMA, Kritski AL. O corpo do portador de tuberculose: enfrentamentos, dificuldades e projeções diárias na terapia ocupacional para obtenção da cura. Rev Pesq: Cuid Fundam Online.2010 [access 2011 Ago 16]; 2(4):1197-213.. Disponible en: http:// www.seer. unirio.br/index.php/cuidadofundamental/article/ view/598/pdf_81
25. Martins SS, Martins TSS. Adesão ao tratamento antirretroviral: vivências de Escolares. Texto Contexto Enferm. 2011; 20(1):111-18.

26. Gargiulo CA, Melo MCSC, Salimena AMO, Bara VMF, Souza IEO. Vivenciando o cotidiano do cuidado na percepção de enfermeiras oncológicas. Texto Contexto Enferm. 2007; 16(4):696-702.

27. Amarante AL, Lepre AS, Gomes JLD, Pereira AV, Dutra VFD. As estratégias dos enfermeiros para o cuidado em saúde mental no Programa Saúde da Família. Texto Contexto Enferm. 2011; 20(1):85-93.

28. Isla Pera P. El cuidador familiar. Una revisión sobre la necesidad del cuidado doméstico y sus repercusiones en la familia. Cultura de los cuidados. 2000; IV(7-8):187-94.

29. De la Cuesta Benjumea C. "Una vida que no es normal": el contexto de los cuidados familiares en la demencia. Index Enfermería. 2011; 20(1-2):41-5.

30. Martorell-Poveda MA, Paz Reverol CL, MontesMuñoz MJ, Jiménez-Herrera MF, Burjalés-Martí MD. Alzheimer: sentidos, significados y cuidados desde una perspectiva transcultural. Index Enfermería. 2010; 19(2-3):106-10.

31. Brullet C. Cambios familiares y nuevas políticas sociales en España y Cataluña. El cuidado de la vida cotidiana a lo largo del ciclo de vida. Educar. 2010; 45: 51-79. 